FORMATION Formation emploi

Revue française de sciences sociales

128 | octobre-décembre 2014

Pêle-Mêle

\title{
Privé ou public : le « choix » du statut d'emploi au sein d'un même groupe professionnel
}

Private of public: the "choice" of employment status within the same occupational group

Privater oder öffentlicher Sektor : die „Wahl“ des Beschäftigungsstatus

innerhalb einer Berufsgruppe

Privado o público : la "elección" del estatuto de empleo dentro de un mismo

grupo profesional

\section{Samuel Julhe et Marina Honta}

\section{CpenEdition}

Journals

Édition électronique

URL : http://journals.openedition.org/formationemploi/4275

DOI : 10.4000/formationemploi.4275

ISSN : 2107-0946

Éditeur

La Documentation française

Édition imprimée

Date de publication : 31 décembre 2014

Pagination : $31-46$

ISSN : 0759-6340

Référence électronique

Samuel Julhe et Marina Honta, « Privé ou public : le « choix » du statut d'emploi au sein d'un même groupe professionnel », Formation emploi [En ligne], 128 | octobre-décembre 2014, mis en ligne le 19 janvier 2015, consulté le 30 octobre 2020. URL : http://journals.openedition.org/formationemploi/ 4275 ; DOI : https://doi.org/10.4000/formationemploi.4275

(C) Tous droits réservés 


\title{
Privé ou public : le « choix » du statut d'emploi au sein d'un même groupe professionnel
}

\author{
SAMUEL JULHE \\ Sociologue, maître de conférences - université de Bordeaux, Laboratoire Cultures, Education, \\ Sociétés (LACES), EA 4140 \\ MARINA HONTA \\ Sociologue, professeure des universités - université de Bordeaux, Laboratoire Cultures, \\ Education, Sociétés (LACES), EA 4140
}

Résumé

Privé ou public : le « choix » du statut d'emploi au sein d'un même groupe professionnel

En France, la démarcation entre salariés de droit privé et agents de la fonction publique est régulièrement mise en débat. Cette recherche s'intéresse aux conditions sociales d'accès à l'un ou à l'autre des statuts dans le cas d'un groupe professionnel, celui des personnels techniques travaillant auprès de fédérations sportives agréées par l'Etat, dont les représentants se partagent entre agents de la fonction publique et salariés de droit privé. Ce travail a été mené principalement par entretiens et suivant une approche par les capacités (capability approach).

\section{Mots clés :}

Champ professionnel, secteur privé, secteur public, métiers du sport, approche par les capabilités

Abstract

Private of public: the "choice" of employment status within the same occupational group

In France, the line between private employees and public servants is regularly debated. This research focuses on the conditions of access to one or other of the status in the case of one specific professional group: the technical staff working with sports federations approved by the state, whose representatives divided between public servants and private employees. This work was carried out by interviews and according to Sen's capability approach.

\section{Keywords:}

Professional field, private sector, public sector, occupation in sport, capability approach Journal of Economic Literature: J 24

Traduction : Auteurs 
En France, la démarcation entre salariés de droit privé et agents de la fonction publique fait figure de summa divisio dans le sens commun. Cela conduit à un débat public récurrent sur les frontières, voire les inégalités, séparant ces deux statuts. La littérature sociologique s'est également appliquée à les comparer, notamment en matière de conditions d'emploi et de travail (Singly \& Thélot, 1988 ; Desmarais \& Abord de Châtillon, 2008 ; Guillemot \& al., 2009 ; Guillemot, 2011). Certains de ces auteurs observent l'activité de salariés du public et du privé au sein d'un même métier (Desmarais \& Abord de Châtillon, 2008 ; Guillemot, 2011). Mais plus rares encore sont les travaux qui s'intéressent précisément aux conditions sociales d'accès à l'un ou à l'autre des statuts, dans le cas d'emplois renvoyant à des fonctions similaires (e.g. enseignants du secondaire exerçant en établissement public ou privé, médecins hospitaliers ou de clinique, ingénieurs des services de l'État ou d'entreprise, etc.). Quid alors des processus présidant à l'intégration d'un individu à un emploi de droit privé ou à la fonction publique, au-delà du rite d'institution que constitue l'obtention d'un concours?

Le présent article invite à questionner ce thème à partir d'une démarche qualitative portant sur un cas singulier, celui des personnels techniques travaillant auprès de fédérations sportives associatives agréées par l'Etat. Le terme d' " entrâ̂neur ", couramment employé pour désigner ces métiers, est aujourd'hui devenu impropre à en décrire le contenu, dans la mesure où le secteur de l'encadrement sportif s'est grandement diversifié. Les temps de face-à-face avec des athlètes ne constituent plus qu'une partie de l'activité à côté de ceux dédiés à la coordination de projets, au développement territorial de l'activité et à son administration (Bernardeau-Moreau \& Bolot, 2009). Leurs tâches peuvent en effet consister à : encadrer des groupes de sportifs depuis l'initiation jusqu'au plus haut niveau de pratique; participer à la détection de la future élite susceptible de concourir lors de rencontres nationales et internationales; développer une ou plusieurs disciplines sportives auprès de publics définis comme prioritaires (e.g. jeunes "des quartiers ", handicapés, etc.) ; préparer les candidats à l'obtention des diplômes délivrés par le ministère chargé des Sports ; etc. À ce jour, ces missions peuvent être assumées par deux types d'acteurs : des Conseillers Techniques Sportifs (CTS), agents de la fonction publique de d'Etat (FPE), et titulaires d'un concours de catégorie A du ministère des Sports, "placés auprès " de fédérations sportives ou de leurs instances territoriales ${ }^{1}$; ou des Conseillers Techniques Fédéraux (CTF), salariés de droit privé embauchés par ces mêmes organisations et dont l'emploi relève de la Convention collective nationale du sport (CCNS) (Pautot, Pautot, 2006). Les uns et les autres ne se distinguent pas fondamentalement par leurs dispositions sociales à l'égard de l'entraînement sportif, ni par leur niveau de diplôme, ni même par les tâches qui leur sont confiées. Partant de ce constat, cette recherche porte sur la mise au jour des caractéristiques des parcours biographiques qui conduisent certains individus à

1. Décrets $n^{\circ} 85-720$ du 10 juillet 1985 et n 2005-1718 du 28 décembre 2005 relatifs à l'exercice des missions de conseillers techniques sportifs auprès des fédérations sportives. 
exercer leur activité sous contrat de droit privé tandis que d'autres accèdent à la fonction publique. Il s'agit ici de dépasser la seule dimension "méritocratique » ou celle du " choix individuel ». In fine, ce travail révèle des enjeux d'attractivité, de recrutement et de gestion des ressources humaines au sein de la fonction publique.

Afin de saisir ce qui relève d'une analyse des conduites de vie, au sens wébérien de lebensfürhung (i.e. manifestations empiriquement observables de l'habitus ou des dispositions sociales) (Colliot-Thélène, 2006, p. 86), dans un contexte de complexification des parcours sociaux (Caradec et al., 2012), nous proposons de suivre une approche par les capacités (capability approach) (Salais, 2007 ; Munck \& Zimmermann, 2008). Elle conduit à s'intéresser au "pouvoir d'une personne de réaliser ou d'être ce à quoi elle accorde de la valeur" (Zimmerman, 2011, p. 111). Plusieurs travaux ont déjà montré son intérêt dans l'analyse de la mobilité professionnelle (Corteel \& Zimmermann, 2007 ; Zimmermann, 2011), de la sécurisation des parcours (Verd \& Vero, 2011 ; Caillaud \& Zimmerman, 2011), mais aussi de l'insertion (Bonvin \& Farvaque, 2007 ; Germain \& Olympio, 2012). Cette approche se propose notamment d'appréhender les accomplissements effectifs des acteurs (e.g. entamer une formation, passer un concours, obtenir un emploi, etc.) au regard des ressources et des droits formels (entitlement) dont ils disposent, mais aussi de l'étendue de leurs capacités (i.e. ensemble des actes et des choix qui peuvent être posés à un moment donné). Autrement dit, il s'agit notamment d'analyser comment différents facteurs sociaux, potentiellement cumulables, conduisent tantôt à élargir tantôt à restreindre les structures d'opportunités auxquelles font face les individus (Salais, 2007, p. 7), ainsi que les mécanismes aboutissant à la réalisation de l'une de ces opportunités. Dans cette perspective, il est central de considérer les options non retenues par les individus mais qui pouvaient l'être. Ainsi, le recours à la notion de capacité, produit de la rencontre entre un individu socialisé et un contexte spécifique, suppose d'aborder l'articulation entre les latitudes de choix individuel, les opportunités de réalisation et un pouvoir d'agir soutenu par des supports collectifs ; cette articulation étant elle-même médiée par des opérateurs de conversion qui permettent la transformation des ressources disponibles (Zimmerman, 2008, p. 119 ; Caillaud \& Zimmerman, 2011, p. 42).

Après avoir décrit la configuration socio-historique qui a conduit à l'émergence d'une cohabitation entre statuts public et privé dans le secteur sportif, une première partie précisera les conditions d'emploi respectives des CTS et CTF. Sera ensuite abordée la reconstruction des parcours conduisant aux carrières de CTS ou CTF. L'orientation " publique " ou " privée " des parcours professionnels sera saisie à l'aune de l'articulation avec le déroulement antérieur d'une carrière amateur dans le secteur sportif d'une part, et d'autre part, de leur configuration familiale au moment où leur est offerte l’opportunité de se présenter au concours. 


\section{Encadré 1 : Méthodologie}

L'encadrement sportif est un secteur d'emploi déjà ancien, toujours en fort développement (Augustin, 2006). II représente près de 100000 emplois en 2011, selon le Centre d'Analyse des Formations, des Emplois, des Métiers dans I'Animation et le Sport (Juliet et al., 2013). Parmi cet ensemble, on distingue : 18000 travailleurs indépendants, 24000 emplois publics, dont 1689 conseillers techniques sportifs (CTS) agents du ministère des Sports; et 58000 éducateurs employés par le mouvement sportif, parmi lesquels il est impossible de séparer les employés des clubs associatifs, majoritaires, et les conseillers techniques fédéraux (CTF) employés par les instances fédérales (comités départementaux, régionaux ou siège fédéral). Ce volume peut néanmoins être estimé à proportion de celui des CTS.

Les données exposées résultent d'une enquête par entretiens semi-directifs, d'une durée moyenne de 1 h 45 , menés auprès de CTS ( 20 ; âgés de 28 à 55 ans) et CTF ( 20 ; âgés de 27 à 57 ans) représentant un large éventail de disciplines sportives : athlétisme, badminton, canoëkayak, football, gymnastique, handball, tennis, judo, karaté, natation, rugby, surf, tennis de table. Ces entretiens ont abordé quatre grandes thématiques : a) les parcours de formation et d'insertion professionnelle ; b) l'explicitation des conditions d'emploi, de travail et du faisceau de tâches réalisé ; c) l'organisation de la vie familiale au regard des contraintes professionnelles ; d) le rapport entretenu à la structure de tutelle (ministère et services déconcentrés pour les CTS vs. fédération et comités territoriaux pour les (TF). Conformément aux préconisations méthodologiques portant sur l'approche par les capacités (Bonvin \& Farvaque, 2007 ; Farvarque, 2008), il s'est agi d'insister, lors des échanges avec les enquêtés, sur la valeur accordée aux différents accomplissements réalisables, mais également d'accentuer le questionnement sur les moments de choix et la différenciation des options jugées accessibles et inaccessibles. Une opération de codage thématique du contenu des entretiens a été effectuée et ce corpus a également donné lieu à la construction d'une série de portraits ( $c f$. annexe sur la version électronique de la revue). Ces entretiens ont été complétés par une exploitation de diverses sources documentaires à des fins de recoupement : rapports publics nationaux, discours ministériels, bulletins syndicaux, textes législatifs et réglementaires relatifs au cadre d'emploi des CTS et CTF.

\section{L'encadrement sportif sous le double regard du public et du privé}

Le modèle d'organisation du sport qui prévaut en France est en bien des points singulier au regard des autres pays européens (Henry, 2009). Construit au sortir de la Seconde guerre mondiale, il repose sur un partenariat entre le mouvement sportif associatif et l'État. Ce dernier entend maintenir un droit de regard sur l'activité des fédérations, tout en leur accordant la responsabilité de gérer une ou plusieurs disciplines sportives ${ }^{2}$. De même, l'encadrement salarié des activités physiques fut, en France, rapidement légiféré, depuis les premières obligations de diplôme qui datent des années 1950, jusqu'à la loi

2. Ordonnance nº5-1922 du 28 août 1945, relative à l'activité des associations, des ligues, fédérations et groupements sportifs. 
de 1963 portant sur l'encadrement des activités physiques ${ }^{3}$, puis la création d'un Brevet d'Etat d'Educateur Sportif (BEES) à trois degrés en 1972 (Loirand, 1996 ; Pierre, 2009) ${ }^{4}$.

La conception d'un service public du sport, qui reste pour une grande part en vigueur aujourd'hui, se diffuse dès le début des années 1960. À partir de cette période, notamment de manière à améliorer le classement de la France parmi les meilleures nations sportives, les fédérations agréées reçoivent une aide en moyens humains. Elle se traduit par la mise à disposition d'agents de l'État experts dans le domaine de la préparation physique et de l'entraînement. Dès le milieu des années 1980, ces professionnels forment le corps des CTS 5 , dont le nombre ne cesse de progresser jusqu'au début des années 1990, pour se stabiliser autour de 1700 agents dans le courant des années 2000. Cependant, depuis plus d'une dizaine d'années, dans une conjoncture générale de réduction des dépenses publiques, et notamment de diminution du nombre de fonctionnaires (Bezès \& Demazière, 2011), se posent les questions du redéploiement des CTS d'une fédération à l'autre, de la réduction globale de leur nombre, voire de la pérennité de ce corps (Lavaure \& Watrin, 2011 ; Honta \& Julhe, 2014).

Suite à l'engagement de la politique de soutien, les fédérations nationales se sont considérablement développées. Elles ont notamment connu un processus de division interne du travail et de salarisation (Bayle, 2010). Plusieurs d'entre elles, parmi les plus importantes (e.g. fédérations de football, tennis, athlétisme, etc.), ont montré des velléités à recruter leurs propres personnels techniques, dénommés de manière générique CTF. Si le recrutement de salariés sous contrat de droit privé est un phénomène ancien dans le mouvement sportif, notamment au sein des clubs, l'intégration de CTF, dévolus au développement de territoires régionaux ou inter-régionaux, s'est accélérée à partir du milieu des années 1990. Ceci s'explique, d'une part, par l'accroissement des ressources issues du secteur marchand dont disposent les fédérations, d'autre part, par l'action de l'État lui-même qui, notamment dans le domaine du sport, a ouvert de nombreux programmes d'aide à l'insertion professionnelle par le recours à des contrats aidés (Honta, 2013). En décidant d'accompagner le développement de l'emploi dans le secteur sportif associatif, plus faible économiquement relativement à d'autres (Tchernonog, 2013), l'État a parallèlement contribué à ce que les CTS ne soient pas les seuls professionnels au sein des instances fédérales à disposer de la licence et du mandat (Hughes, 1996), autrement dit de l'autorisation légitime d'exercer une activité, permettant d'organiser le développement des pratiques sportives et leur encadrement.

3. Loi nº3-807 du 6 août 1963 réglementant la profession d'éducateur physique ou sportif et les écoles ou établissement où s'exerce cette profession.

4. Décret nº $73-490$ du 15 juin 1972 portant création d'un brevet à trois degrés d'éducateur sportif. On distingue ainsi le BEES ler degré, destiné à l'initiation et à l'enseignement général de l'activité, le BEES 2ième degré, portant sur l'entraînement et le perfectionnement technique et le BEES 3ième degré, dévolu à l'expertise et à la recherche.

5. Décrets nº5-720 du 10 juillet 1985 et n²005-1718 du 28 décembre 2005, op. cit. 
Comme pour les CTS, les tâches confiées aux CTF, placés quant à eux directement sous l'autorité hiérarchique des dirigeants sportifs élus (e.g. Président.e d'un comité régional, d'une fédération, etc.), sont très diversifiées. La division du travail entre les deux groupes professionnels n'étant pas fixée par voie réglementaire, des renégociations s'effectuent au cas par cas en fonction des configurations socio-historiques propres à chaque discipline et aux organisations en présence :

"La volonté de la Direction Technique Nationale, c'est de dire que le cadre d'Etat doit gérer le haut niveau. C'est normal que le CTS ait des équipes de France. Et le CTF, c'est normal quil fasse du local, plus le développement et la formation. [...] Moi je ne peux pas moccuper de l'équipe de France, des sélections et faire aussi du développement. On a réussi à mettre en place un partage des tâches avec nos statuts différents. " (Romain, 45 ans, DEUG STAPS - Sciences et techniques des activités physiques et sportives - et BEES 2, CTS)

Comme l'illustre cet extrait d'entretien, il n'est pas rare d'assister à des conflits de juridiction (Abbott, 1988) entre CTS et CTF, i.e. à des oppositions concernant la division du travail entre deux groupes professionnels, les premiers considérant par exemple le niveau national et celui des sportifs de haut niveau comme leur secteur de prédilection, aspects détaillés dans une précédente recherche (Honta \& julhe, 2013)

Par ailleurs, alors même que les tâches effectuées par les uns et les autres sont globalement similaires, de même que leurs conditions de travail - importance du volume de travail (40 à $70 \mathrm{~h}$ par semaine), horaires atypiques et absences répétées du foyer afin d'assurer l'encadrement des athlètes -, les conditions d'emploi des deux types d'acteurs divergent totalement. Le sens commun associe ordinairement le statut de fonctionnaire à une sécurité de l'emploi, mais au prix d'un niveau de rémunération moindre par rapport à un poste équivalent exercé dans le secteur privé, notamment pour les postes de cadre (Guillemot \& al., 2009). Or, au premier abord, les CTS semblent cumuler tous les avantages comparativement à leurs homologues CTF. Au-delà de la sécurité de l'emploi, les CTS perçoivent un traitement aligné sur celui des enseignants d'éducation physique et sportive (EPS) du second degré ${ }^{6}$, tandis que les CTF perçoivent des salaires moins attractifs qui dépendent des grilles établies par la CCNS ${ }^{7}$.

Tous les CTF interviewés rapportent les négociations, toujours serrées, qu'ils doivent mener pour faire valoir leurs prétentions salariales auprès des dirigeants sportifs, mais également leur sentiment de "plafonnement de carrière» (Marc, 2008). Ces négociations, qui renvoient plus largement aux conditions d'emploi et de rémunération observables chez les salariés associatifs (Hély, 2008), sont d'autant plus difficiles qu'il n'existe aucune cohésion syndicale entre les CTF, ce qui les isole face à leur employeur. À ce titre, les rémunérations

6. Comme tout agent de la FPE, les CTS sont rémunérés selon une grille indiciaire. Selon la classe et l'échelon considérés, leur rémunération brute mensuelle varie entre 1600 euros et un maximum de 3600 euros. 7. Selon leur " groupe » d'appartenance et les arguments qu'ils ont pu faire valoir lors de leurs négociations salariales, les CTF perçoivent entre 1400 euros et 2300 euros bruts mensuels. 
des CTF et des CTS sont fréquemment mises en balance par les enquêtés ; la qualité du traitement perçu par les CTS pouvant en effet faire partie des motifs qui orientent vers le concours, notamment pour celles et ceux qui ont exercé des fonctions de CTF antérieurement :

"J'ai persévéré pour le concours. Parce que la stabilisation de l'emploi était une grosse motivation, ça c'est sûr. [...] J'ai pas mal entraîné dans les clubs en complément quand j'étais employé de ligue. Je finissais mon boulot et j'enchaînais dans les clubs le soir, parce quil me fallait un peu plus de rentrées. [...] Pour le salaire entre CTF et CTS, c'est facile, pour moi ç'a a été du simple au double. " (Mathieu, 42 ans, Licence STAPS et BEES 2, CTS)

Dans ce contexte, où les CTS tendent à cumuler les avantages : possibilité d'accéder aux missions les plus prestigieuses, sécurité de l'emploi et meilleurs revenus, tout semblerait pousser les CTF à tenter d'obtenir le concours d'agent de la FPE afin de "sauter le pas et aller du côté des gens de l'autre monde ", comme le résume l'un des enquêtés. Selon les dires de certains acteurs, la raison limitant cet exode serait avant tout le manque de formation des CTF, les empêchant d'atteindre les prérequis à l'inscription au concours. En effet, l'accès au concours permettant de devenir $\mathrm{CTS}^{8}$ exige la possession d'un diplôme de niveau II : une licence en STAPS ou un Brevet d'Etat d'Educateur Sportif (BEES) du 2ième degré ou un Diplôme d'Etat Supérieur de la Jeunesse, de l'Education Populaire et du Sport (DESJEPS).

"Souvent, ceux qui sont CTF, ils n'ont que le BE1. Et entre le brevet d'Etat premier degré et le brevet d'Etat deuxième degré et le concours de prof de sport, il y a quand même une petite marche. Et souvent, s'ils ont fait CTF, je ne veux pas les dénigrer, mais c'est souvent un peu par défaut, parce que scolairement... C'est pas par choix, c'est très peu par choix. [...] Pour être CTS, il faut quand même avoir une formation un peu généraliste et ce n'est pas donné à tous ceux qui sont CTF. » (Éléonore, 32 ans, master STAPS et BEES 2, CTS)

Or, cet argument semble relever de l'idée reçue. Les filières de formation initiale qui conduisent aux fonctions de CTS et de CTF sont aujourd'hui très proches. Les uns et les autres sont généralement titulaires d'un diplôme délivré soit par le ministère de l'Enseignement supérieur et de la Recherche - filière en STAPS -, soit par le ministère chargé des Sports - BEES 1er ou 2ième degré, désormais remplacés par le Brevet professionnel de la jeunesse de l'éducation populaire et du sport (BPJEPS) et le Diplôme d'Etat de la jeunesse, de l'éducation populaire et du sport (DEJEPS) ${ }^{9}$. Ainsi, la grande majorité des CTF interviewés (17/20) disposent des prérequis permettant de s'inscrire au concours,

8. Le taux de sélectivité de ce concours est relativement élevé : 8,7\% en 2010 (49 postes pour 564 candidats) ; 7,3\% en 2009 (54 postes pour 738 candidats) ; 6,1\% en 2008 (69 postes pour 1128 candidats).

9. $C f$. Décret n²001-792 du 31 août 2001 portant règlement général du Brevet professionnel de la jeunesse, de l'éducation populaire et du sport délivré par le ministère de la Jeunesse et des Sports ; Décret $\mathrm{n}^{\circ}$ 20061418 du 20 novembre 2006 portant règlement général du diplôme d'Etat de la jeunesse, de l'éducation populaire et du sport, délivré par le ministère chargé de la Jeunesse et des Sports. 
mais seule une minorité (5/20) s’y est déjà présentée. Suivant l'approche par les capacités (Farvarque, 2008), il s'agit d'observer respectivement comment CTS et CTF en activité ont pu valoriser l'accès au fonctionnariat lors de leur phase de formation, mais aussi d'appréhender quels opérateurs de conversion participent à l'actualisation des structures d'opportunités de leur parcours professionnel.

\section{2) Devenir CTS : 2 l'agencement propice des séquences biographiques}

Exercer professionnellement dans le domaine du sport plonge ses racines dans un long parcours entamé de façon précoce, durant l'enfance ou l'adolescence, avec une spécialisation très rapide dans une discipline sportive. L’entrée dans le métier de CTS résulte d'un long processus d'incorporation d'un goût pour les activités physiques, le plus souvent initié par le père et/ou un frère, et d'acculturation au monde sportif comme pratiquant.e, puis compétiteur ou compétitrice ; ce processus est suivi du réajustement d'une vocation pour les métiers de l'encadrement des activités physiques, selon des mécanismes similaires au secteur de la culture où un loisir vécu comme une "passion " (e.g. théâtre, cinéma, musique, etc.) peut donner lieu à un souhait de professionnalisation (Dubois, 2013). L'extrait suivant résume cet enchainement :

"Mon cheminement, il est simple. Ma rencontre avec l'activité s'est faite dans l'enfance. Vers 13 ou 14 ans, ça s'est accéléré avec des compétitions au national. J'ai intégré Sport Etude [...] Pour la fac, j'ai fait une licence STAPS, option entrainement, naturellement. [...] Déjà, pendant les études, j'avais commencé à entraîner. J'avais pensé bosser dans un club et puis, entretemps, sest présentée pour moi l'opportunité du professorat, donc, je me suis investi sur la préparation du concours, et tout s'est enchainé comme ça." (Martial, 49 ans, licence STAPS, et BEES 2, CTS)

La pratique sportive donne lieu au développement d'une carrière amateur (Chevalier \& Coinaud, 2008 ; Gojard \& Terral, 2009), i.e. à l'accumulation d'expériences et de reconnaissance au sein d'un univers de pratique tout d'abord abordé en tant que loisir, mais également à des cursus scolaires particuliers pour une bonne part des CTS interviewés $(13 / 20)$ (e.g. sport-étude, classe à horaires aménagées, etc.). Dès le lycée, se fait jour le souhait de pouvoir encadrer leur activité de prédilection, que ce soit à titre professionnel ou amateur. Cette socialisation sportive précoce conduit les CTS interviewés à privilégier des études dans le domaine des STAPS (18/20). Si le professorat d'éducation physique est fréquemment évoqué comme premier souhait d'orientation professionnelle (15/20), les stages en établissement scolaire précipitent l'arrêt de ce projet. Ils conduisent à un repli sur un public "qui a envie d'apprendre » et sur les métiers de l'entraînement et de la performance. À ce titre, les enquêtés entament, parallèlement à leurs études supérieures, des formations aux diplômes délivrés par le ministère des Sports, avant tout perçus comme un 
" sésame professionnel ", mais aussi un complément à la "culture " acquise à l'université, dont les diplômes sont jugés trop "généralistes " (Lima \& Mossé, 2010). C'est entre 18 et 25 ans que les CTS interviewés débutent leurs premières tâches d'entraînement en club, tout en poursuivant leur carrière sportive en compétition.

Dans ce contexte mêlant études supérieures, rejet du métier d'enseignant d'EPS, poursuite de la pratique compétitive et première approche de l'entrainement, se redéfinit progressivement leur structure d'opportunités en matière d'horizon professionnel. Les entretiens biographiques révèlent une faible connaissance préalable des métiers de l'entrainement ; seuls deux des enquêtés visaient le concours du professorat de sport dès l'entrée de leurs études, l'un ayant un parent enseignant d'EPS impliqué dans l'entrainement d'une équipe sportive en club et l'autre un père entraîneur. Dans la majorité des cas (15/20), les futurs CTS ont été sollicités et encouragés à passer ce concours par des membres de ce corps de la FPE. Ce processus de "repérage " et de " cooptation " s'effectue le plus souvent au cours des stages dédiés à la préparation au BEES ou en marge de compétitions, et est à l'initiative de CTS déjà en place, d'un entraineur national (EN), voire du directeur technique national (DTN) lui-même :

"C'est dans mon activité de compète que j'ai connu des CTS. J'étais dans les stages régionaux, dans les stages inter-régionaux. Ensuite, j'avais été sollicitée dans les formations. Donc voilà, j'ai eu le pied à l'étrier... On m'a encouragée : "Vas-y, il faut y aller". Il y en a un qui m'a dit: "Je te verrais bien dans ce métier là, tu devrais passer le concours." [...] Nous mêmes, quand on sent quelqu'un qui est dans cette optique là, on lui dit qu'il peut y avoir cette filière. " (Fanny, 27 ans, master STAPS et BEES 2, CTS)

Cet extrait d'entretien parmi d'autres montre comment cette étape de " repérage " par des acteurs spécifiques du mouvement sportif, qui peuvent être envisagés comme des opérateurs de conversion, contribue à ouvrir la structure d'opportunités des individus et à les orienter vers la fonction publique en leur donnant des gages d'élection.

Pour autant, la présence de ces cautions oratoires ne suffit pas à opter pour le concours et le métier de CTS. Lorsque l'opportunité du professorat se présente, les individus semblent davantage accepter, par anticipation, les contraintes liées au métier de CTS (importance du volume de travail, horaires atypiques, etc.) s'ils ne sont pas investis sur le plan conjugal et familial. En effet, même s'il peut conduire à un gain de rémunération, tenter d'obtenir ce concours revient à accepter l'idée d'une mobilité géographique, les nouveaux lauréats se voyant proposer par leur DTN un choix d'affectation entre deux ou trois régions. De même, cela exige d'être en accord avec un rythme de travail ponctué par de très fréquents déplacements - selon les missions auxquelles ils sont affectés, les CTS peuvent être absents de leur foyer entre 50 et 250 nuits par an -, dont l'influence est majeure sur la conduite de la vie domestique des CTS, comme cela a pu être montré par ailleurs (Julhe \& Honta, 2012). 
"Au début, j’hésitais pour le concours et puis, je l'ai tenté à un moment où jétais libre de tout mouvement [i.e. suite à une rupture conjugale] [...] J'ai reçu l'avis d'affectation un vendredi par courrier, il était pour le jour même. J'appelle la Direction régionale qui me dit: "Vous avez le temps, lundi c'est bon!" [...] J'ai rien pu anticiper et j'ai dù déménager en catastrophe [...] En fait, c'est quand même un métier formidable, si on n'est pas sédentaire à tout prix et pas trop centrée sur soi et son couple. (Emilie, 35 ans, licence STAPS et BEES 2, CTS)

Le propos d'Emilie montre en quoi la configuration familiale (e.g. être en couple, avoir des enfants, etc.) est en soi un opérateur de conversion des ressources dont disposent les individus. En effet, une "vacance " sur ce plan contribue à une possible focalisation sur l'univers professionnel et à une mobilisation des ressources disponibles favorisant le choix de se présenter au concours. En définitive, l'analyse des parcours d'insertion professionnelle des CTS décrit des individus cumulant un ensemble de ressources qui s'agencent par ailleurs selon une temporalité propice à leur conversion en nouveaux accomplissements professionnels.

\section{Rester CTF pour privilégier la sphère familiale}

Les récits biographiques des CTF révèlent, comme chez leurs homologues du secteur public, un engagement précoce dans le secteur sportif. Leur carrière amateur se déroule également à travers l'accès aux modalités compétitives de leur discipline de prédilection. Comme les CTS, ils privilégient des études en STAPS, mais dans une moindre proportion (14/20). Les scolarités apparaissent plus chaotiques, ponctuées de redoublements ou de réorientations $(11 / 20)$, même si leur niveau de diplôme le plus élevé se révèle finalement similaire à celui des CTS.

Contrairement aux CTS, seule une minorité des CTF interviewés (3/20) indique avoir été sollicitée par des acteurs du service public du sport (e.g. CTS, EN, DTN, etc.), lors de la phase de formation initiale. Ils font cependant eux aussi l'objet d'un processus de « repérage ", mais l'opérateur de conversion des ressources sportives est ici incarné par les élus des instances fédérales. En "récompense " d'un engagement de longue date au sein d'un club et de leurs années de bénévolat, un poste d'entraineur rémunéré leur est fréquemment proposé au terme de la validation de leur BEES $1^{\text {er }}$ degré $(16 / 20)$ :

"Pour mon premier poste, j’ai eu de la chance. Comme jétais impliqué avant dans le club en tant que bénévole, j'ai été poussé par des parents qui voyaient mon investissement dans le club. Ils ont dit: "Il est là, il sinvestit, donc sil a envie du poste, pourquoi pas le lui donner !". " (Michel, 34 ans, DEUG STAPS et BEES 2, CTF)

Comme dans le cas des CTS, un processus de distinction et d'élection (Zimmermann, 2011) - une reconnaissance par les pairs fondée sur des affinités électives - est à l'œuvre, 
mais il se réalise ici à un niveau local, permettant un premier ancrage professionnel dans la région où l'entraîneur s'est formé ou a effectué ses études. Ce n'est que dans un second temps, alors qu'ils ont poursuivi leur formation et entamé leur vie professionnelle, qu'ils seront informés de l'opportunité d'obtenir le concours de CTS. Faute d'avoir obtenu les gages provenant des acteurs publics du sport, et notamment de CTS en poste, certains tendent à s'auto-exclure de la voie du concours. Par ailleurs, alors qu'ils sont déjà entrés en fonction sous contrat de droit privé, les CTF perçoivent avec davantage d'acuité ce qu'ils considèrent comme l'une des contraintes majeures du métier de CTS : l'obligation de mobilité ( $c f$. supra). Implantés sur un territoire où ils ont déjà entamé leur vie professionnelle, il devient plus délicat d'envisager le déracinement.

"J'avais la possibilité de devenir CTS, mais mon souhait, c'était de travailler ici. Je n'ai pas eu l'ambition d'aller voir ailleurs... J'aurais pu me lancer là-dedans, mais là aussi il y a des contreparties, ça veut dire qu'il faut être mobile, être capable d'aller n'importe où... Je pense qu'au niveau familial, on a fait un choix et je ne le regrette pas. [...] Il y en a plein qui passent par là, mais on avait 30 ans et deux enfants. Donc, on a préféré qu'ils grandissent ici. Quand on n'a pas le choix, on fait ce que l'on vous propose, mais moi j'estime que j'ai eu la chance de pouvoir choisir. (Hervé, 51 ans, licence STAPS et BEES 2, CTF)

Ceci est encore exacerbé lorsqu'ils ont déjà fondé un foyer, comme le souligne l'extrait précédent, l'obtention du concours obligeant à un déménagement familial. De ce fait, nombre d'entre eux préfèrent conserver leur statut de CTF et ne sont plus véritablement en capacités de se présenter au concours qui, bien quaboutissant à un poste améliorant leur situation financière et sécurisant leur emploi, viendrait déstabiliser la stratégie résidentielle établie en famille. Davantage que les CTS, ils prêtent attention à leurs conditions de travail (e.g. déplacements, horaires, etc.) et souhaitent ne pas se "laisser déborder par le métier».

"Le concours, jy ai pensé, mais j'ai envie de dire "trop tard". Il était hors de question que je fasse quoi que ce soit tant que mes gamines étaient en bas âge. C'était hors de question. De par le boulot déjà, parce que t’es jamais là. [...] Donc je métais dis : "tant quelles ne sont pas ado... terminé quoi". Je ne conçois pas de déménager tout le monde. Donc ça serait faire quelque chose maintenant qu'elles sont grandes. Sauf que, maintenant, à 40 balais, passer le concours, je ne sais pas si j'en aurais la capacité intellectuelle et l'envie, tout en bossant. [...] Et puis, ça voudrait dire aller où? Parce que quand t'as un train de vie installé et compagnie. Donc, je me dis que c'est un peu tard. " (Vanessa, 40 ans, licence STAPS et BEES 2, CTF)

Ce type d'arbitrage entre vie familiale et ambition de développement professionnel peut être illustré par un dernier exemple qui souligne l'importance des agencements des temps biographiques sur les choix de carrière :

"On mavait fait passer des messages lors de la formation au BE2, pour passer le professorat [...] Lors de l'oral, on me dit: "Bon, normalement, on n'a pas le droit de te le dire, mais il faut que tu passes le professorat". [...] Plusieurs personnes mont dit : "Il faut que tu y ailles". [...] 
Mais actuellement, je suis là avec toute la famille. Si jai le concours, je fais quoi? Comme une amie qui est passée CTS, hop, Ille-de-France. [...] Non, déjà pendant deux ans, ma compagne et moi on a vécu à 300 kilomètres l'un de l'autre, alors non. " (Nicolas, 39 ans, DEUG sciences économiques et BEES 2, CTF)

Dans le cas présent, l'intérêt pour le concours de la fonction publique est préservé grâce aux sollicitations récurrentes émanant de CTS en poste, maintenant la structure d'opportunité ouverte et réactualisant ainsi l'idée qu'une carrière dans ce secteur reste envisageable et souhaitable. En somme, le parcours des CTF se caractérise, comparativement à celui de leurs homologues de la fonction publique, par une moindre visibilité initiale auprès de représentants nationaux du mouvement sportif, mais également par une plus grande attention portée à la sphère domestique au moment où leur est donnée la possibilité de franchir le cap du concours. Là encore, l'agencement des séquences biographiques et l'ordonnancement de l'acquisition des ressources disponibles importent davantage que leur volume global pris à un instant donné.

\section{Conclusion}

Si l'obtention d'un diplôme de niveau II (e.g. licence, BEES $2^{\circ}$ degré, DESJEPS, etc.) place les individus étudiés en capacité de se présenter au concours du professorat de sport, tous ne font pas ce choix. Opter pour la fonction publique ou pour le maintien dans le secteur privé lors de la phase d'insertion et le début du parcours professionnel relève d'abord de l'agencement des séquences biographiques qui composent le parcours antérieur. La reconstruction des parcours de formation et d'insertion des représentants des deux groupes abordés met au jour des séquences idéal-typiques. Les CTS se trouvent majoritairement pris dans une configuration où s'enchaînent carrière sportive amateur de haut niveau, études supérieures, choix de se présenter au concours du professorat de sport, obtention du concours et consolidation de la carrière professionnelle, puis consolidation de la vie familiale. Dans le cas des CTF, le modèle se présente davantage en une séquence qui juxtapose carrière sportive amateur de haut niveau, études supérieures, premier emploi et consolidation de la vie familiale, opportunité de se présenter au concours du professorat de sport, refus de cette opportunité afin de maintenir la configuration familiale et professionnelle présente.

Ce travail illustre également en quoi deux individus disposant au premier abord de ressources et de droits identiques (entiltement) ne sont pas nécessairement en capacité de s'en saisir de la même façon. Les ressources ne peuvent se traduire en libertés réelles d'action, faute d'une possibilité de les convertir. Cette assertion, a priori triviale, recouvre néanmoins des enjeux importants, notamment en matière d'attractivité et de recrutement au sein de la fonction publique. Afin d'attirer les «meilleurs talents » vers le fonctionnariat, comme le préconisent les réformes de l'action publique en matière de gestion des ressources humaines (IGEP, 2008), il ne suffit pas de multiplier les prérequis à la présentation d'un concours ou d'élever les exigences des épreuves. Il convient également de lutter 
contre le biais de sélection marquant à la fois les individus qui se présentent aux concours et ceux qui s'en auto-excluent. À ce titre, "l'approche par les capacités ne vise pas à dénoncer l'existence de ces contraintes (tâche à la fois facile et peu féconde), mais à évaluer dans quelle mesure les réformes en direction de l'État social actif augmentent (ou diminuent) le degré de contrainte imposé aux individus et permettent (ou entravent) une répartition plus équitable des limitations et restrictions imposées par toute vie en société» (Bonvin \& Farvarque, 2007, p. 14).

Dans le cas des CTF et des CTS, on peut par exemple penser qu'une meilleure prise en compte des conditions de travail (horaires atypiques, fréquence des déplacements, etc.) et l'adjonction d'aménagements portant sur l'articulation entre vie professionnelle et vie familiale (Pailhé \& Solaz, 2009 ; Julhe \& Honta, 2012), à travers notamment un accompagnement lors des phases de mobilité géographique ou une aide à la garde des enfants, favoriseraient la diversification des profils des candidats, notamment en direction d'une féminisation ${ }^{10}$. Faute de quoi, l'attribution du concours ne reflète plus seulement une dimension méritocratique mais résulte également d'une sélection négative où seuls se présentent les individus acceptant par anticipation de supporter les contraintes du métier exercé dans le cadre public.

Dans le cas des CTS, cela revient " uniquement " à mettre en jeu la préparation de futures gloires sportives, mais tout porte à croire que ce type de question se pose également dans d'autres secteurs. Ainsi, l'accès au métier d'enseignant du second degré est périodiquement questionné, sans pour autant que soit abordé le " choix " d'enseigner en établissement public ou privé (Berger \& D’Ascoli, 2011) ; la seconde alternative pouvant pourtant permettre de pallier la nomination dans une zone géographique non désirée (Pochard, 2008). Le problème se pose de façon assez proche pour les médecins, chez qui l'orientation statutaire réalisée en fin d'étude (e.g. médecin hospitalier ou non, libéral ou salarié, etc.) se révèle cruciale pour le déroulement de leur début de carrière mais aussi pour leurs choix résidentiels et familiaux (Robelet et al., 2006). Cela pose enfin la question de la cohabitation de personnes réalisant des tâches similaires, voire identiques, mais disposant de statuts différents, comme cela est fréquemment le cas aujourd'hui dans les établissements hospitaliers (Schweyer, 2009).

\section{Bibliographie}

Abbott A. (1988), The system of professions. An essay on the division of expert labor, Chicago, The University of Chicago Press.

Augustin J.-P. (dir.) (2006), Vers les métiers de l'animation et du sport : la transition professionnelle, Paris, La Documentation française.

10. En 2014, $16 \%$ des CTS sont des femmes, contre $30 \%$ chez les CTF. 
Bayle E. (2010), « La gouvernance des fédérations d'associations chargées d'une mission de service public : le cas des fédérations sportives françaises ", Politiques et management public, 27, (1), pp. 121-142.

Bernardeau-Moreau D., Bolot F. (2009), « Du technicien au gestionnaire : regard sur les mutations de la filière sportive territoriale ", Revue française d'administration publique, 131, pp. 583-594.

Berger J.-L., D’Ascoli Y. (2011), «Les motivations à devenir enseignant : revue de la question chez les enseignants de première et deuxième carrière ", Revue française de pédagogie, 175, pp. 113-146.

Bezès P., Demazière D. (dir.) (2011), "New Public Management et professions dans l'État : au-delà des oppositions, quelles recompositions ", Sociologie du travail, 53, (3), pp. 293-348.

Bonvin J.-M., Farvaque N. (2007), « L'accès à l'emploi au prisme des capabilités, enjeux théoriques et méthodologiques ", Formation Emploi, 98, pp. 9-22.

Caillaud P., Zimmerman B. (2011), « Sécurisation des parcours et liberté professionnelle : de la "flexicurité" aux capacités ", Formation Emploi, 113, pp. 33-48.

Caradec V., Ertul S., Melchior J.-P. (2012), Les dynamiques des parcours sociaux, Rennes, PUR.

Chevalier V., Coinaud C. (2008), "Carrières d'amateurs et cursus universitaires : le sens multiple des parcours des étudiants en STAPS », in Cart B., Giret J.-F., Grelet Y., Werquin P., Derrière les diplômes et certifications, les parcours de formation et leurs effets sur les parcours d'emploi, Paris, Céreq, pp. 179-189.

Colliot-Thélene C. (2006), La sociologie de Max Weber, Paris, La Découverte.

Corteel D., Zimmermann B. (2007), "Capacités et développement professionnel ", Formation emploi, 98, pp. 25-39.

Cour des comptes, 2013, Sport pour tous et sport de haut niveau : pour une réorientation de l'action de l'État, Rapport thématique.

Desmarais C., Abord de Châtillon E. (2008), « Existe-t-il encore des différences entre le travail des managers du public et ceux du privé ", Revue française d'administration publique, 128, pp. 767-783.

Dubois V. (2013), La culture comme vocation, Paris, "Raison d'agir ».

Farvarque N. (2008), «Faire surgir des faits utilisables. Comment opérationnaliser l'approche par les capacités ? ", in Munck (de) J., Zimmermann B. (dir.), La liberté au prisme des capacités. Amartya Sen au-delà du libéralisme, Paris, Ed. de l'EHESS, pp. 51-80. 
Germain V., Olympio N. (2012), " Parcours scolaires en France et espace d'opportunités : une analyse à l'aune de la théorie des capabilités de Sen ", Formation Emploi, 120, pp. 13-33.

Gojard L., Terral P. (2009), "Insertion professionnelle des étudiants STAPS de la filière entraînement : modification du rapport au sport et intellectualisation ", in BernardeauMoreau D., Colinet C. (dir.), Les éducateurs sportifs en France depuis 1945. Question de professionnalisation, Rennes, PUR, pp. 151-176.

Guillemot D. (2011), "Travail dans le public et le privé : une intensification parallèle », Travail et Emploi, 128, pp. 23-40.

Guillemot D., Jeannot G., Peyrin A. (2009), «Travail du public, travail du privé : similitudes et différences. Premiers apports de l'enquête "changement organisationnel et informatisation" ", Revue française d'administration publique, 132, pp. 789-803.

Hély M. (2008), «À travail égal, salaire inégal : ce que travailler dans le secteur associatif veut dire ", Sociétés contemporaines, 69, pp. 125-148.

Henry I. (2009), "European Models of Sport : Governance, Organisational Change and Sports Policy in the EU”, Hitotsubashi Journal of Arts and Sciences, 50, (1), pp. 41-52.

Honta M. (2013), «Les associations sportives et les politiques publiques d'aide à l'insertion professionnelle ", Diversité. Ville-École-Intégration, 171, pp. 180-185.

Honta M., Julhe S. (2013), " Les professions du secteur public saisies par la privatisation. Le cas des conseillers techniques sportifs ", Gouvernement et action publique, 2, (1), pp. 63-87.

Honta M., Julhe S. (2014), « La rénovation de la gestion des carrières dans le secteur public à l'épreuve des relations entre État et groupes d'intérêt. Les cas des Conseillers Techniques Sportifs du ministère des Sports ", Politiques et Management Public, 31, (2), pp. 239-257.

Hughes E.C. (1996), Le regard sociologique. Essais choisis, Paris, Éd. de l'ÉHESS.

IGPED (2008), Performance de la fonction ressources humaines : définitions et cadres d'analyse, rapport remis au ministère du Budget, des Comptes publics et de la Fonction publique.

Juillet N., Buisine S., Gouju J.-L. (2013), Panorama des situations professionnelles du champ des activités physiques et sportives, Rapport remis au CAFEMAS, p. 94.

Julhe S., Honta M. (2012), "L'articulation travail-famille chez les conseillers techniques sportifs : situations asymétriques entre hommes et femmes ", Sociologie, 4, (3), pp. 341-357.

Lavaure Ph., Watrin D. (2011), Rapport relatif aux missions des cadres techniques sportifs placés auprès des fédérations sportives, Paris, Inspection générale de la Jeunesse et des Sports. Lima L., Mossé Ph. (dir.) (2010), Le sport comme métier? Les STAPS des études à l'emploi, Toulouse, Octarès. 
Loirand G. (1996), Une difficile affaire publique : une sociologie du contrôle de l'Etat sur les APS et sur leur encadrement professionnel, Thèse de $3^{\text {ième }}$ cycle, sociologie, université de Nantes.

Marc D. (2008), «Le plafonnement des carrières : le cas des techniciens de l'environnement, entre contrainte réglementaire et manque de reconnaissance ", Politique et management public, 26, (2), pp. 99-110.

Munck J., Zimmermann B. (2008), "Qu'est-ce qu'une capacité ? ", in Munck (de) J., Zimmermann B. (dir.), La liberté au prisme des capacités. Amartya Sen au-delà du libéralisme, Paris, Éd. de l'EHESS, pp. 21-50.

Pailhé A., Solaz A. (dir.) (2009), Entre famille et travail. Des arrangements de couple aux pratiques des employeurs, Paris, La Découverte.

Pautot S., Pautot M. (2006), La convention collective nationale du sport, Paris, PUS.

Pierre J. (2009), « Des brevets d'Etat d'éducateur sportif aux diplômes professionnels de 1963 à nos jours ", in Bernardeau-Moreau D., Colinet C. (dir.) Les éducateurs sportifs en France depuis 1945. Question sur la professionnalisation, Rennes, PUR, pp. 109-150.

Pochard M. (2008), Livre vert sur l'évolution du métier d'enseignant, Rapport remis au ministre de l'Education nationale.

Robelet M., Lapeyre N., Zolesio E. (2006), Les pratiques professionnelles des jeunes générations de médecins. Genre, carrière et gestion des temps sociaux, Rapport remis au Conseil National de l'Ordre des Médecins.

Salais R. (2007), "À la recherche de principes novateur pour l'action publique ", Formation Emploi, 98, pp. 5-8.

Schweyer F.-X. (2007), « Santé, contrat social et marché : la fonction publique hospitalière en réformes ", Revue française d'administration publique, 132, pp. 727-744.

Singly F., Thélot C. (1988), Gens du privé, gens du public : la grande différence, Paris, Dunod.

Tchernonog V. (2013), Les paysage associatif français, Paris, Dalloz.

Verd J.-M., Vero J. (2011), « Pourquoi la flexicurité mérite-t-elle qu’on débatte à partir de l'approche par les capacités ?», Formation Emploi, 113, pp. 5-14.

Zimmermann B. (2008), "Capacités et enquête sociologique ", in Munck (de) J., Zimmermann B. (dir.), La liberté au prisme des capacités. Amartya Sen au-delà du libéralisme, Paris, Éd. de l'EHESS, pp. 113-137.

Zimmermann B. (2011), Ce que travailler veut dire. Une sociologie des capacités et des parcours professionnels, Paris, Economica. 